\title{
UTILIZAÇÃO DOS ALERTAS DE CONTROLE COMO FERRAMENTA PARA A FIDELIZAÇÃO DA CLIENTELA DE PEDIATRIA EM UM AMBULATÓRIO PÚBLICO
}

\section{USE OF CONTROL ALERTS AS A TOOL FOR THE LOYALTY OF THE CUSTOMERS OF PEDIATRICS IN A PUBLIC CLINIC}

\section{USO DEL CONTROL DE ALERTAS COMO UNA HERRAMIENTA PARA LA LEALTAD DE LOS CLIENTES DE PEDIATRÍA EN UNA CLÍNICA PÚBLICA}

\author{
Sonia Francisca Monken \\ Universidade Nove de Julho \\ soniamonken@uninove.br \\ Regina Célia Batista Moreno \\ Universidade Nove de Julho \\ reginabmoreno@hotmail.com
}

\author{
Submetido em: 23/11/2014 \\ Correções obrigatórias: 15/12/2014 \\ Artigo aceito em: 20/02/2015
}

\section{RESUMO:}

O absenteísmo às consultas de pediatria no serviço público tem comprometido o atendimento à demanda, acarretando o desequilíbrio do sistema de agendamento devido à reutilização do fluxo de atendimento. Em pediatria, na maioria dos casos atendidos, necessita de controle mensal, o não comparecimento à consulta interfere na resposta do diagnóstico, no esquema terapêutico e na qualidade de vida dessas crianças. O Serviço Social promove o retorno à consulta evitando que se permaneça muito tempo sem atendimento médico ou sem medicação. Buscaram-se, como solução, alertas de controle (red flags) no sistema de agendamento desencadeando providências em engajar a mãe no comparecimento às consultas. O trabalho foi desenvolvido na especialidade de Neonatologia de Recém Nascido de Alto Risco (NRNAR) onde a taxa de absenteísmo no mês de abril estava em $38 \%$, e após a implantação dos alertas de controle no mês de maio, reduziu para $11 \%$. Fica visível a redução do absenteísmo mediante a utilização dos alertas de controle pelo sistema de agendamento, e a intervenção do Serviço Social para conscientização da mãe visando à fidelização dessa clientela.

Palavras chaves: Absenteísmo. Fidelização. Alertas de controle

\begin{abstract}
:
Absenteeism in the specialty of Pediatrics at a public clinic has compromised meet demand, leading to an imbalance in the system schedule due to the return flow of such faulty customer service. In addition, it is known that this specialty, served in most cases requires a monthly control, and not attending the consultation response interferes with the diagnosis, therapeutic scheme and quality of life of these children. The Social Services intervention is in the sense that these children have a faster return preventing remain long without medical attention and even without medication. It was sought as a solution, control alerting, and a kind of red flags in the scheduling system triggering actions of social workers to engage the mother in attending medical appointments. The work was developed in the art of high-risk newborns where the absenteeism rate in April was 38\%, and after the implantation of control alerting (red flags) in May, decreased to $11 \%$. Is visible the reduction of absenteeism using red flags by the scheduling system, and intervention of a Social Service to raise awareness of the mother seeking the loyalty of this clientele.
\end{abstract}

Keywords: Absenteeism. Loyalty. Alert control, Red Flags

\section{RESUMEN:}

El absentismo a consultas de Pediatría en el servicio público ha comprometido la atención de la demanda, conduce al desequilibrio sistema programación debido a la reutilización del flujo de servicio. En Pediatría, en la mayoría de los casos se reunió, requiere control mensual, la falta de asistencia a la consulta en la respuesta de la diagnosis, interfiere en el esquema terapéutico y la calidad de vida de estos 
niños. Servicios sociales promueve un retorno para evitar consulta permanecen demasiado tiempo sin atención médica o medicamentos. Buscó una solución, control de alertas (bandera roja) en la programación de sistema de disparo acción en participar a la madre en la asistencia a las citas. El trabajo se desarrolló en la especialidad de neonatología del recién nacido de alto riesgo (NRNAR) donde la tasa de absentismo en el mes de abril fue de $38 \%$, y después de despliegue de las alertas de control en el mes de mayo, reducido a $11 \%$. Llega a ser visible la reducción del absentismo laboral mediante el uso de control de alertas mediante la programación de sistema y la intervención de Servicios Sociales para la conciencia de la madre a la lealtad de los clientes.

Palabras clave: absentismo laboral. Fidelización de clientes. Alertas de controle

\section{INTRODUÇÃO}

A gestão das operações dos sistemas de saúde brasileiro enfrenta constantemente dilemas de insuficiência de recursos e variabilidade de distribuição de serviços médicos e hospitalares especializados. Podendo resultar, por conseguinte, na má qualidade da assistência, seja por não dar acesso à população aos tratamentos necessários ou pela falta de continuidade da atenção à saúde. Além desses fatores restritivos depara-se com o absenteísmo, entendido como a falta do paciente ao tratamento ou consulta agendada (CAVALCANTI et al, 2013).

O absenteísmo às consultas médicas de um ambulatório público tem comprometido o atendimento à demanda de pacientes, acarretando um desequilíbrio no sistema de agendamento, especificamente nas especialidades pediátricas, quando a clientela faltosa retorna em outra data para reagendamento de novas consultas.

Além do absenteísmo e reagendamento serem atividades restritivas ao fluxo ambulatorial, tem-se o fator de que a maioria dos pacientes necessita de um acompanhamento mensal para uma melhor qualidade de vida no futuro.

No ambulatório estudado, a oferta de vagas para primeiras consultas é planejada para o público externo na proporção de $70 \%$ e $30 \%$ para atender a demanda interna da instituição, respectivamente, uma vez que o ambulatório pertence a um complexo hospitalar público. Os retornos são agendados pessoalmente, após consulta médica, através de filipeta fornecida pelo médico. O sistema de agendamento não realiza marcação de consulta por telefone.

Um levantamento realizado nesse ambulatório de especialidades, pertencente a um complexo hospitalar público, no município de São Paulo, no período de Fevereiro a Abril de 2014, apontou que o nível de absenteísmo nas consultas de retorno das especialidades de pediatria, estava acima de 19\%, compreendendo as consultas em neuropediatria (20\%), clínica de recém-nascido (RN) de alto risco $(32 \%)$, pneumologia (16\%) e moléstias infectocontagiosas (10\%).

O Serviço Social, como integrante da equipe multidisciplinar do ambulatório, pesquisou alternativas no sentido das crianças terem um retorno mais rápido, independente do agendamento pelos responsáveis, com isso evitando que permaneçam sem atendimento médico e até sem medicação.

Sendo assim, buscou-se como solução deste entrave, o desenvolvimento de alertas de controle (red flags) no sistema de agendamento, que irá apontar as crianças faltosas, mediante relatórios semanais, e desencadeará providências das assistentes sociais em engajar os responsáveis no comparecimento às consultas de pediatria.

O objetivo deste relato técnico é apresentar a aplicabilidade dos alertas de controle (red flags) como ferramenta institucional que permita melhorar a fidelização da 
clientela infantil ao tratamento proposto pelo profissional médico e consequente redução do nível de absenteísmo.

\section{REFERENCIAL TEÓRICO}

A ausência dos usuários do Sistema Único de Saúde (SUS) às consultas e procedimentos marcados previamente, o absenteísmo, é uma das causas das perdas e desperdícios dos recursos, não só pelo aumento da demanda como também pelo retorno do usuário ao sistema de operações assistenciais (SANTOS, 2008).

Nas especialidades pediátricas, o absenteísmo, além de causar repetição das ações do sistema de agendamento, traz reflexos significativos para a saúde e desenvolvimento da clientela, pois o seguimento sistematizado permite a detecção precoce de desvios da normalidade. No caso especial do RN de alto risco egresso de Unidade de Terapia Intensiva Neonatal - UTIN, o absenteísmo provoca descontinuidade no controle do crescimento pondero estatural, do desenvolvimento neuropsicomotor, da avaliação da visão e audição, sendo fator de falha no acompanhamento clínico, possibilitando outras reinternações e, portanto, onerando os custos hospitalares (MEIO et al., 2005).

Parte do gerenciamento das operações em serviços de saúde está baseada no conceito de regulação. CAVALCANTI et al (2013) apontam para a discussão acerca da regulação, como sendo a formulação e implementação de estratégias que possibilitem o acesso adequado aos serviços de atenção à saúde. Sendo na adequação dos recursos assistenciais (oferta) e nas necessidades de atenção e de assistência à saúde da população (demanda).

Os autores acrescentam que a regulação da atenção à saúde compreende a contratação, a regulação do acesso à assistência ou regulação assistencial, o controle assistencial, a avaliação da atenção à saúde, a auditoria assistencial e as regulamentações da vigilância sanitária. Englobando o conjunto de tecnologias e logística que norteiam as relações de demanda dos usuários dos serviços de saúde. Dessa forma, pode-se entender a regulação como gestão de serviços enquanto assume as funções de direção, controle, organização e adaptação do sistema de prestação de assistência à saúde (Figura 1).

Figura 1. Ideias fundamentais e relacionadas aos conceitos de regulação.

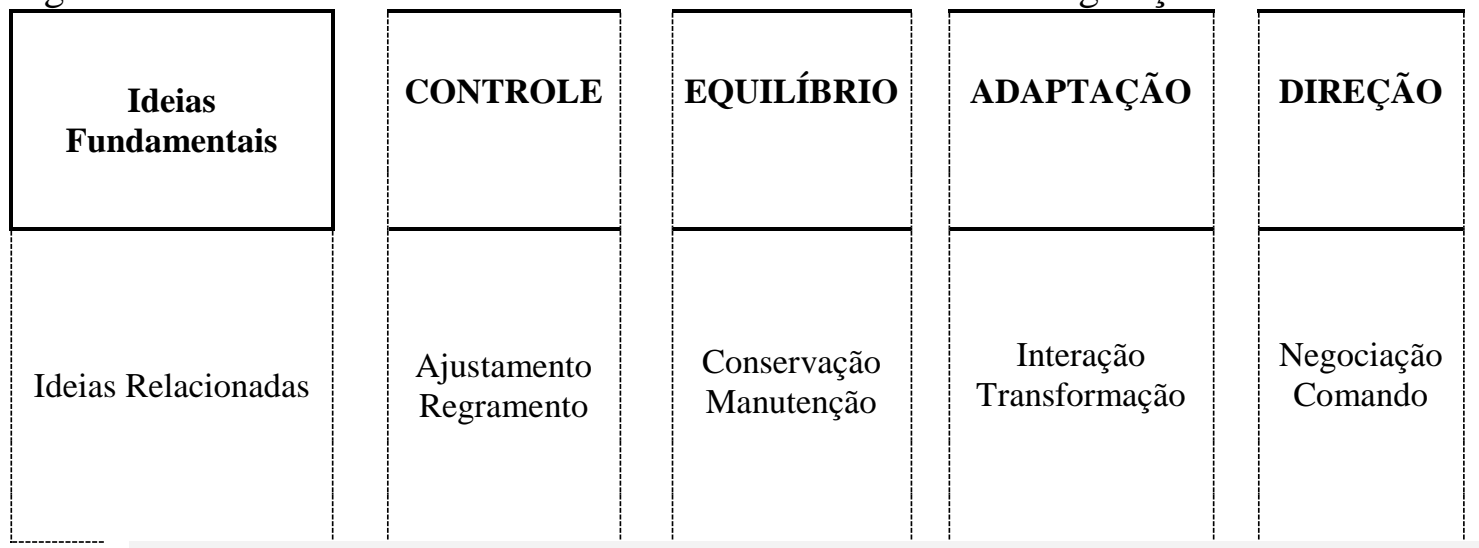

Fonte: Oliveira RR e Elias PEM, 2012 - Conceitos de regulação em saúde no Brasil. Revista de Saúde Pública, 46(3), 571-576. 
O monitoramento do absenteísmo na pediatria é entendido, neste estudo, como instrumento de regulação do setor, na melhoria na qualidade dos serviços prestados, pois assume um papel de estabelecer padrões de qualidade e segurança nas prestações dos serviços de saúde, melhorando as condições de tratamento e cura de inúmeras doenças que cada usuário do SUS é acometido (Conselho Nacional De Secretários De Saúde [CONASS, 2007]),

Cresce a exigência para que os serviços de saúde organizem-se de modo a responder às necessidades das pessoas e ofereçam um cuidado efetivo e humanizado, provendo todas as informações que o usuário necessita (ESCRIVÃO, 2007).

Assim sendo, a intervenção do Serviço Social é no sentido de conscientizar os responsáveis dos RNs quanto à necessidade do acompanhamento regular e contínuo até a alta concedida pelo profissional médico.

Adiciona-se a este panorama a questão da capacidade de obter, processar e compreender informações e serviços básicos de saúde, necessários para tomar decisões pertinentes sobre sua própria saúde e sobre cuidados médicos, denominado de Letramento em Saúde (LS). O LS inadequado é prevalente e se associa a desfechos clínicos indesejáveis que impactam desfavoravelmente na história natural de várias doenças. A sobrecarga de informações novas a serem seguidas contribui e, consequentemente, desencadeiam desfechos clínicos negativos. Comparado aos indivíduos com LS adequado, aqueles com LS inadequado apresentam menor aderência medicamentosa, irregularidade de visitas médicas, diagnóstico tardio, pior qualidade de vida, custos médicos mais elevados e consequentes menores eficácia de uso dos serviços de saúde, além de maiores taxas de hospitalização e mortalidade (SANTOS et al., 2012).

A falta à consulta e a não aderência ao tratamento sinaliza para o profissional médico o não entendimento por parte dos responsáveis da necessidade da aderência ao tratamento proposto ao $\mathrm{RN}$ de alto risco.

Estudos anteriores mostraram que ao implementar a estratégia de alerta ao usuário, como por exemplo, a de confirmação de presença de usuário por telefone, na antevéspera à cirurgia eletiva, foi possível reduzirem $30 \%$ os casos de absenteísmo cirúrgico. Desse modo, a confirmação do usuário à cirurgia eletiva sinalizou que o cancelamento cirúrgico em decorrência do absenteísmo do usuário pode ser considerado uma causa evitável, já que os usuários que não compareceram tinham essa informação na véspera da cirurgia (DE AVILA; BOCCHI, 2013).

A função de controle do sistema se estabelece através da auditoria como um processo sistemático para obter evidências sobre a conformidade das afirmações a critérios estabelecidos, precisa desenvolver-se dentro de uma abordagem estruturada. $\mathrm{Na}$ sua fase de pré-planejamento é importante a apreciação de potenciais pontos de alerta, usualmente denominados pelos auditores como red flags (CUPERTINO; MARTINEZ, 2009).

Os alertas de informações ou red flags são sinalizadores pré-determinados e de conhecimento do gestor para tomada de decisão, detectando o mais cedo possível, as informações que caracterizam uma mudança do meio ambiente da empresa, seja ela qual for, tecnológica, comercial, industrial, política ou jurídica.

Para tanto, não é necessário que a empresa desenvolva um sistema de observação panorâmico, como descrito como campo do monitoramento tecnológico. 
(GRIMES; SCHULZ, 2005; MARIN, 2010), mas podendo-se focar em uma área específica da produção do serviço.

O Serviço Social é responsável pela avaliação dos riscos sócio-familiares, suporte para as famílias, orientação quanto aos direitos previstos em lei para as crianças portadoras de deficiências, orientação quanto às instituições de auxílio social e aos encaminhamentos para instituições de reabilitação. É responsável também pela detecção dos pacientes que abandonam o acompanhamento e pela recuperação dos mesmos, relacionando os motivos prováveis de abandono do tratamento (MEIO et al., 2005).

Nas ações socioassistenciais o Serviço Social intervém na solução quanto ao atendimento (facilitar marcação de consultas), no não entendimento do tratamento indicado e falta de condições para realizá-lo devido ao preço do medicamento prescrito, do transporte urbano necessário para o acesso ao serviço de saúde, ou horário de tratamento incompatível com o horário de trabalho dos usuários (Conselho Federal de Serviço Social [CFESS] 2008).

No ambulatório de especialidades, a intervenção do Serviço Social deu-se como forma de resgatar a fidelização da clientela de $\mathrm{RN}$ de alto risco, tendo em vista que são crianças que podem apresentar futuras patologias se não forem acompanhadas com frequência, e foi a partir dessa observação técnica que se realizou o levantamento do absenteísmo nas especialidades pediátricas.

\section{ASPECTOS METODOLÓGICOS}

O estudo baseou-se no método de pesquisa documental, que se caracterizam pelo estudo de diversos documentos, em específico os manuais de procedimentos, relatos técnicos, estatísticas de atendimento e relatórios gerenciais de um complexo hospitalar público, sendo as análises dos dados expressos por frequência absoluta e relativa ao total da amostra, sem a aplicação de testes estatísticos específicos.

Utilizou-se da estratégia de pesquisa-ação, que possibilitou ao mesmo tempo o estudo do fenômeno e a proposta de resolução de um problema (MARTINS; THEÓFILO, 2009).

Como proposta do método de pesquisa-ação os autores puderem ter interação entre as pessoas envolvidas na situação investigada, a equipe multiprofissional pôde discutir e sugerir prioridades de intervenção para o Ambulatório de Especialidades. .

O Ambulatório de Especialidades faz parte do complexo hospitalar, composto de: hospital geral de nível terciário referência em politraumas; hospital psiquiátrico; laboratório de análises clínicas e um centro de referência do idoso, está situado no Município da Cidade de São Paulo - SP, pertence à Secretaria de Estado de Saúde de São Paulo, atendendo a clientela de 06 distritos de saúde, totalizando uma população de 2.200.000 habitantes, sendo referência também para outros Municípios vizinhos. Realizou no ano de 2013 a média de 11.500 consultas/mês, sendo que 30\% desse total referem-se à primeira consulta.

É caracterizado como Ambulatório público de nível terciário, nas especialidades clínicas, cirúrgicas, equipe multiprofissional e serviços de diagnóstico e terapia. $\mathrm{O}$ horário de funcionamento é das 7 horas às 19 horas, de segunda à sexta-feira, e em alguns sábados acontecem mutirões para reduzir a demanda reprimida de algumas especialidades como: cardiologia, neurologia, otorrinolaringologia. 
Está sediado em um prédio de 03 andares, onde se instala a estrutura assistencial com Centro Cirúrgico e 34 consultórios médicos (Tabela 1, Tabela 2).

Tabela 1 - Estrutura Ambulatorial de Pediatria

\begin{tabular}{ll}
\hline Especialidades & Número de Consultórios \\
\hline Neurologia & 1 \\
\hline Cardiologia & 1 \\
\hline Gastroenterologia & 1 \\
\hline Pneumologia & 1 \\
\hline RN de alto Risco & 1 \\
\hline Moléstias infectocontagiosas & 1 \\
\hline Endocrinologia & 1 \\
\hline TOTAL & 7 \\
\hline
\end{tabular}

Fonte: Registros Internos do Complexo Hospitalar - 2014

Tabela 2 - Estrutura Ambulatorial Geral

\begin{tabular}{|c|c|c|}
\hline Especialidade & Especialidades Atendidas & $\begin{array}{l}\text { Número de } \\
\text { Consultórios }\end{array}$ \\
\hline Oftalmologia & - & 01 \\
\hline Clínica médica & $\begin{array}{l}\text { Cardiologia } \\
\text { Endocrinologia } \\
\text { Pneumologia } \\
\text { Neurologia } \\
\text { Infectologia } \\
\text { Gastroenterologia } \\
\text { Otorrinolaringologia } \\
\text { Dermatologia } \\
\text { Proctologia } \\
\text { Urologia }\end{array}$ & 08 \\
\hline Clínica cirúrgica & $\begin{array}{l}\text { Cirurgia vascular } \\
\text { Neurocirurgia adulta e } \\
\text { infantil } \\
\text { Cirurgia geral } \\
\text { Cirurgia plástica } \\
\text { Cirurgia pediátrica }\end{array}$ & 11 \\
\hline Ginecologia & $\begin{array}{l}\text { Climatério } \\
\text { Mastologia } \\
\text { pré-natal de alto risco } \\
\text { Planejamento familiar } \\
\text { Colposcopia } \\
\text { Histeroscopia } \\
\end{array}$ & 04 \\
\hline Ortopedia & - & 03 \\
\hline TOTAL & & 27 \\
\hline
\end{tabular}

Fonte: Registros Internos do Complexo Hospitalar - 2014

O Ambulatório possui mais 19 consultórios voltados para a equipe multidisciplinar no atendimento de Psicologia adulta e infantil, Serviço Social, Terapia Ocupacional, Buco maxilo facial, Odontologia, Acupuntura e Medicina do Trabalho. Além dos procedimentos de Reiki e Cromoterapia, salas de gesso, salas de curativos, ostomias, sala de cateterismo vesical, sala de tratamento assistido voltado aos pacientes 
portadores de hepatites crônicas B e C em tratamento medicamentoso e sala de medicação.

As salas de espera estão localizadas nos corredores laterais dos andares, e no primeiro andar tem uma brinquedoteca idealizada pelo Serviço de Voluntariado do Hospital.

Os serviços prestados na área de serviços de estomaterapia é referência de atendimento no sistema público, com uma média de atendimento de 400 pacientes mensais.

A área de serviços de diagnóstico e terapia oferecem exames de eletrocardiograma, eletroencefalograma, eletroneuromiografia, espirometria, nasofibroscopia, bera e urodinâmica, realizando cerca de 753 exames/mês. Além dos exames de imagem, tais como: densitometria óssea, raios-X, ultrassonografias, mamografia, ecodoplercardiograma e audiometria, são realizados no andar térreo, pela parceria da Secretaria de Estado da Saúde (SES) e Organização Social de Saúde (OSS).

O Centro Cirúrgico está situado no subsolo do Ambulatório, possui 04 salas cirúrgicas e 04 de Recuperação Pós Anestésica (RPAs), realiza uma média de 209 cirurgias mensais, biópsias hepáticas e de próstata.

O serviço de agendamento está localizado no andar térreo do Ambulatório e é realizado através da Central de Regulação de Ofertas de Serviços de Saúde, sendo o contrato gerenciado diretamente pela Secretaria de Estado da Saúde (SES), conta com um balcão para informações e distribuição de senhas e 10 guichês para atendimento.

Os guichês são categorizados para atendimento de senhas preferenciais e não preferenciais, lista de espera e abertura de Ficha de Atendimento Ambulatorial (FAA). Possui ainda uma central telefônica para contatar pacientes que aguardam em lista de espera. Na média, realizam 600 agendamentos/dia, entre consultas para esta unidade e para demais unidades referenciadas no sistema de agendamento.

As vagas são distribuídas conforme critérios estabelecidos entre SES e Diretoria Regional de Saúde I - DRS I. Das vagas de primeira consulta são oferecidas $70 \%$ para DRS I e $30 \%$ são para atender a demanda interna. Os retornos são agendados pessoalmente. $\mathrm{O}$ sistema de agendamento emite comprovante com dados de consultas e exames, incluindo o preparo de procedimento, endereço da unidade executante e ponto de referência.

Os pacientes de primeira consulta chegam até o Ambulatório com comprovante de agendamento e encaminhamento da unidade solicitante, dirigem-se à recepção do térreo, abrem a FAA e são encaminhados aos respectivos andares das especialidades, onde receberão uma senha e orientação para aguardar em frente ao consultório de sua especialidade.

Os pacientes de retorno dirigem-se ao respectivo andar, recebem senha e orientação para aguardar em frente ao consultório da especialidade. Estes já possuem FAA ou prontuários. Lembrando que os prontuários são solicitados ao Núcleo de Arquivo Médico com 48 horas de antecedência.

Após consulta médica, são orientados nos balcões de pós-atendimento quanto a exames, encaminhamentos para outros especialistas, retornos na especialidade e em seguida direcionados ao setor de agendamento. $O$ sistema de marcação de consultas não realiza agendamentos por telefone. Os retornos são agendados pessoalmente após consulta, com filipeta fornecida pelo médico especialista. Quando o paciente não comparece à consulta agendada necessita de nova filipeta, exclusivamente emitida pelo 
médico, devendo comparecer ao Ambulatório no dia e horário de atendimento do profissional.

Como descrito anteriormente, o Ambulatório mantém 30 especialidades dentre elas a pediatria (crianças de 0 a 12 anos), que apresenta uma oscilação no gerenciamento da demanda, a partir do absenteísmo às consultas. Esse tipo de gargalo na operação de serviços de consultas médicas provoca um desnivelamento na capacidade produtiva do Ambulatório.

Foi feito um levantamento nas bases de dados do agendamento ambulatorial, buscando os pacientes que não compareceram às consultas no período de Fevereiro à Abril de 2014, o período escolhido retrata uma preocupação da equipe médica e da administração do ambulatório.

Constatou-se que o absenteísmo às consultas de retorno das especialidades de neuropediatria, pneumologia, infectologia pediátrica e $\mathrm{RN}$ de alto risco apresentaram uma média de $19 \%$ de ausência. (Tabela 3)

Tabela 3

Especialidades Pediátricas com Total de Consultas Agendadas, Atendidas e Absenteísmo Referentes aos Meses de Fevereiro, Março e Abril de 2014.

\begin{tabular}{|c|c|c|c|c|c|c|c|c|c|}
\hline 2014 & Neurol & & Pneum & ologir & & Infecto & & RN de & isco \\
\hline \multirow[b]{2}{*}{ Fevereiro } & $\begin{array}{l}\text { Agend } \\
\text { Absent }\end{array}$ & Atend & Agend & Aten & Absent & $\begin{array}{l}\text { Agend } \\
\text { Absent }\end{array}$ & Atend & $\begin{array}{l}\text { Agend } \\
\text { Absent }\end{array}$ & Atend \\
\hline & $\begin{array}{l}130 \\
\mathbf{2 2 \%}\end{array}$ & 101 & 109 & 92 & $16 \%$ & $\begin{array}{l}42 \\
10 \%\end{array}$ & 38 & $\begin{array}{l}25 \\
\mathbf{2 8 \%}\end{array}$ & 18 \\
\hline Março & $\begin{array}{l}118 \\
\mathbf{1 7 \%}\end{array}$ & 98 & 102 & 90 & $13 \%$ & $\begin{array}{l}38 \\
12 \%\end{array}$ & 30 & $\begin{array}{c}20 \\
\mathbf{3 0 \%}\end{array}$ & 14 \\
\hline Abril & $\begin{array}{l}167 \\
\mathbf{2 1 \%}\end{array}$ & 121 & 127 & 97 & $19 \%$ & $\begin{array}{l}51 \\
8 \%\end{array}$ & 46 & $\begin{array}{c}24 \\
\mathbf{3 8 \%}\end{array}$ & 15 \\
\hline
\end{tabular}

Fonte: Estatística Ambulatorial por Especialidade Pediátrica - 2014

Sabe-se que essas especialidades, na maioria dos casos atendidos são crianças que necessitam de um controle mensal, e que o não comparecimento à consulta interfere na resposta do diagnóstico, no esquema terapêutico e na qualidade de vida dessas crianças. São doenças que uma vez bem tratadas quando criança, com certeza será um adulto com melhor qualidade de vida. Portanto, a intervenção do Serviço Social é no sentido de que essas crianças tenham um retorno mais rápido, independente da mãe ou responsável estar agendando, visando evitar que permaneçam muito tempo sem atendimento médico e sem a medicação de controle.

Sendo assim, surge a necessidade de criar alternativas que viabilizem com mais rapidez a detecção dessa clientela, o que resultou como proposição de solução deste entrave, a criação de alertas de controle (red flags), no sistema de agendamento, que irá apontar as crianças faltosas, mediante relatórios semanais, e desencadeará providências das assistentes sociais em engajar a mãe ou responsável no comparecimento às consultas.

A sinalização do absenteísmo é de grande importância e essencial para as especialidades que necessitam de tratamento contínuo. Os alertas de informações ou red flags são sinalizadores pré-determinados e de conhecimento do gestor para tomadas de decisão (GRIMES; SCHULZ, 2005; MARIN, 2010). 
Para implementar os alertas de controle no sistema de agendamento do ambulatório em estudo, o suporte tecnológico se torna essencial. A definição de como esse sinalizador deve ser, porém, muitas vezes é uma tarefa morosa, e a adequação do sistema de agendamento torna-se trabalhosa devido a falta de pessoal no atendimento.

Figura 2. Relatório de beneficiários assinalados red flag

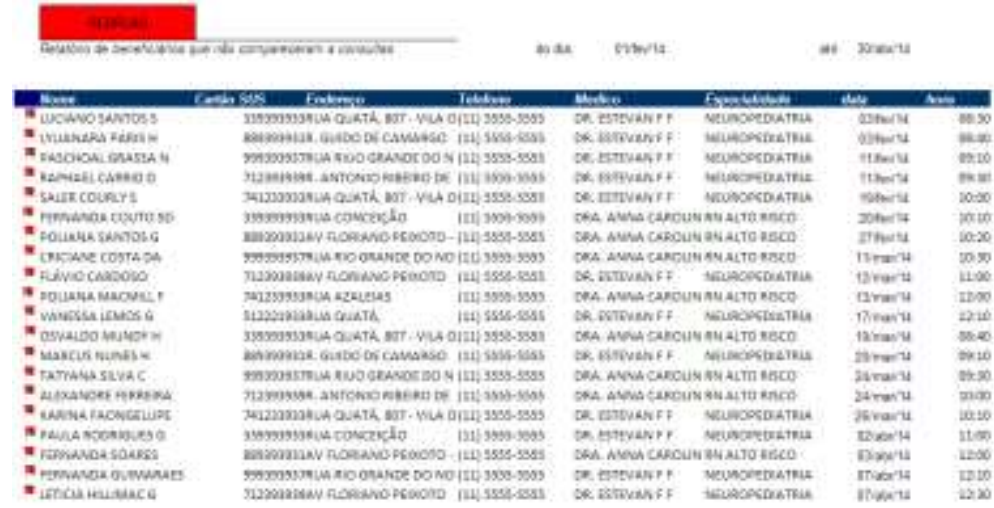

Fonte: Sistema de Acompanhamento Serviço Social ${ }^{1}$

As Assistentes Sociais mediante relatório contendo nome, telefone de contato, (Figura 2) farão a abordagem por meio telefônico, primeiramente questionando sobre a razão da falta, em seguida esclarecendo sobre a necessidade do acompanhamento pelo especialista e por fim perguntando se há interesse em continuar com o acompanhamento no Ambulatório. Caso a resposta seja positiva, será comunicada a data de nova consulta. O sistema de agendamento, através da central telefônica, realizará novo contato 48 horas que antecede à consulta com intuito de confirmação de presença, evitando com isso o desperdício da vaga.

${ }^{1}$ A tela mostra dados fictícios mantendo a confidencialidade dos envolvidos - usuários e médicos

\section{ANÁLISE DOS RESULTADOS}

A especialidade de RN de Alto Risco apresentou a maior média de absenteísmo (32\%) em relação às demais especialidades no período de análise, na especialidade são ofertadas em torno de 27 vagas de retorno mensalmente. O Relatório de Beneficiários Faltantes (Figura 2) permitiu o contato com os responsáveis por 14 beneficiários faltantes, 13 deles tiveram com resposta positiva à continuidade do tratamento no Ambulatório, 01 responsável pelo paciente preferiu continuar o atendimento com o pediatra da Unidade Básica de Saúde - UBS próxima de sua residência, apenas 01 paciente não foi possível o contato devido o número de telefone estar incorreto, mesmo assim, o endereço registrado permitiu o contato telefônico com a UBS pertencente à região de domicílio, sendo solicitado uma visita domiciliar informando nova data de consulta e a atualização dos telefones para um contato futuro.

Os demais agendamentos foram efetuados, conforme disponibilidade da agenda médica, sendo os quatorze pacientes distribuídos no período de 13/05/14 à 29/05/14. Os registros de consultas confirmam que neste período das 14 vagas de retorno, 11 foram confirmados os retornos em consulta. Todos os responsáveis foram contatados pela central telefônica do sistema de agendamento para confirmação da consulta. Nesse mesmo período foram agendadas 26 consultas de retorno e 23 pacientes compareceram, 
apresentando um índice de $11 \%$ de absenteísmo, evidenciando-se uma queda média de $21 \%$ no índice de absenteísmo no período para a especialidade. (Tabela 4)

Tabela 4:

Números de consultas agendadas e atendidas na especialidade de RN de alto risco no Ambulatório em estudo no período de fevereiro a maio de 2014.

\begin{tabular}{lcccc}
\hline Mês & Agendados & Atendidos & \multicolumn{2}{c}{ Absenteísmo } \\
\hline & & & $\mathrm{n}$ & $\%$ \\
Fevereiro/14 & 25 & 18 & 07 & 28 \\
Março/14 & 20 & 14 & 06 & 30 \\
Abril/14 & 24 & 15 & 09 & 38 \\
Maio/14 & 26 & 23 & 03 & 11 \\
\hline
\end{tabular}

Fonte: Estatística Ambulatorial 2014

Os dados da Tabela 4 mostram que o absenteísmo dos meses estudados foram respectivamente, $28 \%, 30 \%$ e $38 \%$ ausências mensais, e após a implantação do alerta de controle com o objetivo de identificar as crianças faltantes para a intervenção do Serviço Social, adicionada a ação de contato telefônico pela central telefônica do agendamento 48 horas que antecede à consulta para confirmação da presença, o absenteísmo atingiu o percentual de mês de $11 \%$, apresentando redução de $21 \%$ no índice de absenteísmo.

Embora, o Ministério da Saúde não tem definido um parâmetro do que seria uma taxa máxima do absenteísmo aceitável (CAVALCANTI et al., 2013), sabe-se que nessas especialidades, a maioria dos casos atendidos necessita de um controle mensal e que o não comparecimento à consulta interfere na resposta do diagnóstico, no esquema terapêutico e na qualidade de vida das crianças.

\section{CONCLUSÕES}

As ações de redução do absenteísmo às consultas de RN de alto risco no Ambulatório de Especialidades permite melhorar a assistência, melhorar a qualidade de vida dessa clientela, evitar futuras internações, uma vez que essas crianças serão acompanhadas mensalmente, e em havendo necessidade com retornos mais frequentes.

A intervenção propicia provável redução de gastos dos recursos públicos na medida em que o paciente não participa reincidentemente do sistema assistencial, da mesma forma a introdução de tecnologia de processos contribui para a segurança do paciente e da equipe multidisciplinar envolvida, possibilitando melhorias e elevando a qualidade da assistência (SANTOS, 2008; DA SILVA; BARBOSA, 2014).

$\mathrm{O}$ absenteísmo na especialidade de pediatria RN de alto risco é um problema expressivo que traz consequências consideráveis para a qualidade de vida dessas crianças no período em que foi realizada a pesquisa, a redução do absenteísmo contribuiu para a melhoria do acompanhamento desses pacientes.

A necessidade de se criar alternativas capazes de aperfeiçoar e melhorar a qualidade na assistência e na gestão de serviços de saúde deve ser uma constante em todos os estabelecimentos de saúde, a ferramenta de relatórios de red flag permite reunir informações anteriormente desconectadas do fenômeno, tornando-as públicas e consistentes em relatórios gerenciais, possibilitando seu acesso de maneira transparente 
e de livre acesso à equipe profissional para tomada de decisão e intervenção (FERRAZ et al., 2014).

Por meio deste estudo fica visível o impacto da redução do absenteísmo mediante a utilização dos alertas de controle (red flags) pelo sistema de agendamento e a intervenção do Serviço Social para a conscientização dos responsáveis pelos pacientes pediátricos visando à fidelização dessa clientela.

Infere-se que ações conjuntas e inserção de novas ferramentas são de fundamental importância para minimizar a taxa de absenteísmo e oferecer um atendimento mais humanizado.

Os autores reconhecem as limitações do estudo, como a sua realização por um curto período de tempo e com um número reduzido de pacientes. Entretanto, o desenvolvimento de processos de gerenciamento de absenteísmo às especialidades pediátricas que envolvam um maior número de pacientes e por um período mais abrangente, apresentando as intervenções do Serviço Social na condução da fidelização dos responsáveis aos tratamentos, aprofundamentos das questões tecnológicas de informação e ampliação das pesquisas de fatores de adesão aos tratamentos pediátricos serão decisivos na diminuição do absenteísmo às consultas pediátricas, no equilíbrio da capacidade produtiva ambulatorial do sistema público de saúde e da maior fidelização aos tratamentos, com melhoria nas relações de custos e qualidade dos sistemas de saúde públicos.

\section{REFERÊNCIAS BIBLIOGRÁFICAS}

CAVAlCANTI, R. P., CAVAlCANTI, J. C. M., SERRANO, R. M. S. M., DE SANTANA, P. R. Absenteísmo de consultas especializadas nos sistemas de saúde público: relação entre causas e o processo de trabalho de equipes de saúde da família, João Pessoa-PB, Brasil. Tempus Actas de Saúde Coletiva, v. 7, n. 2, p. 63, 2013.

CONSELHO FEDERAL DE SERVIÇO SOCIAL (CFESS). Série: Trabalho e Projeto Profissional nas Políticas Sociais. Brasília - DF. Parâmetros para atuação de Assistentes Sociais na política de Saúde (2008), p. 40-41.

CONSELHO NACIONAL DE SECRETÁRIOS DE SAÚDE (CONASS). Brasília, 2007. p. 74. Coleção para entender o SUS,

CUPERTINO, C. M.; MARTINEZ, A. L. Qualidade da auditoria e earnings management: risk assessment através do nível dos Accruals Discricionários. Contabilidade Vista \& Revista, v. 19, n. 3, p. 69-93, 2009.

DA SILVA, N. D. M., BARBOSA, A. P. Implantação de Sistema de Notificação Eletrônica de Tecnologias Em Saúde. RAHIS v 11, no. 4. 2014.

DE AVILA, M. A. G.; BOCCHI, S. C. M. Confirmação de presença de usuário à cirurgia eletiva por telefone como estratégia para reduzir absenteísmo. Revista da Escola de Enfermagem da USP, v. 47, n. 1, p. 193-197, 2013. 
DE OLIVEIRA, R. R., ELIAS, P. E. M. Conceitos de regulação em saúde no Brasil. Revista de Saúde Pública, v. 46, n. 3, p. 571-576, 2012.

ESCRIVÃO, Á. Uso da informação na gestão de hospitais públicos. Ciência \& Saúde Coletiva, v. 12, n. 3, p. 655-666, 2007.

FERRAZ, R. R. N., QUONIAM, L. AlVARES, L. M. A. R.. Avaliação de redes multidisciplinares com a ferramenta scriptlattes: os casos da nanotecnologia, da dengue e de um programa de pós-graduação Stricto Sensu em Administração. Encontros Bibli: Revista Eletrônica de Biblioteconomia e Ciência da Informação 19.40: 67-98, 2014.

GRIMES, D. A., SCHULZ, K. F. Case-control studies: research in reverse. The Lancet v.359, no. 9304 p. 431-434, 2005.

MARIN, F. H. Sistemas de Informação em Saúde: Considerações Gerais. Journal of Health Informatics - ISSN 2175-4411, 2010.

MEIO, M., MAGlUTA, C., DE MELlO, R. R., MOREIRA, M. E. L. Análise situacional do atendimento ambulatorial prestado a recém-nascidos egressos das unidades de terapia intensiva neonatais no Estado do Rio de Janeiro. Ciência \& Saúde Coletiva, v. 10, p. 299-307, 2005.

SANTOS, J. S. Absenteísmo dos usuários em consultas e procedimentos especializados agendados no SUS: um estudo em um município baiano. 2008. Mestrado. Universidade Federal da Bahia - UFBA - Instituto de Saúde Coletiva. Programa de Pós Graduação em Saúde Coletiva, Vitória da Conquista - BA. Recuperado de https://repositorio.ufba.br/ri/handle/ri/6759, acessado em 22/04/2014.

SANTOS, L., MANSUR, H. N., PAIVA, T. F., COLUGNATI, F. A., BASTOS, M. G. Letramento em saúde: importância da avaliação em nefrologia; Health literacy: importance of assessment in nephrology. Jornal Brasileiro de Nefrologia, v. 34, n. 3, p. 293-302, 2012.

THEÓPHILO, C.R., MARTINS, G. DE A. Metodologia da Investigação Científica para Ciências Sociais Aplicadas. $2^{\mathrm{a}}$ ed. Editora Atlas. p. 55, 2009. 